\title{
Acute Effects of Metrological Factors on Cerebrovascular Diseases among Type2 Diabetes Patients: case crossover evidence from Beijing, China
}

\author{
Deginet Aklilu" ${ }^{1,2 *}$, Zhiwei Li ${ }^{1,2 *}$, Tianqi Wang ${ }^{1,3}$, Wei Feng ${ }^{1,2}$, XiaLi4, Lixin Tao ${ }^{1,2}$, Wei Wang ${ }^{5}$, Moning Guo ${ }^{3}$, \\ Yanshuang Jiang ${ }^{1}$, Anteneh Tamirat ${ }^{6}$, Xiangtong Liu ${ }^{1,2 \star}$ and Xiuhua Guo ${ }^{1,2 \star}$ \\ ${ }^{1}$ Department of Epidemiology and Health Statistics, School of Public Health, Capital Medical University, Beijing, 100069,China. \\ ${ }^{2}$ Beijing Municipal Key Laboratory of Clinical Epidemiology, Capital Medical University, Beijing, 100069,China. \\ ${ }^{3}$ Beijing Municipal Commission of Health and Family Planning Information Center,China. \\ ${ }^{4}$ Department of Mathematics and Statistics, La Trobe University, Melbourne, Victoria, Australia. \\ ${ }^{5}$ Global Health and Genomics, School of Medical Sciences and Health, Edith Cowan University, Perth, Western Australia, Australia. \\ ${ }^{6}$ Department of Pharmacology, College of Medicine and Health Sciences, Wachemo University, Ethiopia. \\ "DeginetAklilu and Zhiwei Li contributed equally to this work.
}

Asian Journal of Complementary and Alternative Medicine. Volume 10 Issue 01

Published on: 28/02/2022

*Author for Correspondence: Xiuhua Guo, School of Public Health, Capital Medical University, No.10 Xitoutiao, You'anmenWai, Fengtai District, Beijing 100069, China; Fax: +86-010-83911508, Tel.: +86-010-83911508; E-mail: statguo@ccmu.edu.cn

Xiangtong Liu, School of Public Health, Capital Medical University, No.10 Xitoutiao, You’anmenWai, Fengtai District, Beijing 100069, China; Fax: +86-010-83911508, Tel.: +86-010-83911508; E-mail: xiangtongl@ccmu.edu.cn

Cite this article as: Aklilu D, Li Z, Wang T, Feng W, Li X, Tao L, et al. Acute Effects of Metrological Factors on Cerebrovascular Diseases among Type2 Diabetes Patients: case crossover evidence from Beijing, China. Asian Journal of Complementary and Alternative Medicine, Vol 10(1), 20-29:2022.

\begin{abstract}
Background: Acute effects of metrological factors on cerebrovascular diseases (CBVD) have become a growing public health concern. However, the impact of those factors on CBVD among type 2 diabetes (T2D) patients has not been well studied in the study area. The main aim of this study was to assess the acute effects of metrological factors including temperature, relative humidity and atmospheric pressure on incidence of CBVD among type 2 diabetes patients in Beijing, China.

Methods: Data was obtained from 258 large general hospitals from the Beijing Public Health Information Center Cardiovascular Case Database and China. Meteorological Administration covering 16 districts in Beijing from 2014 to 2018 . Time-stratified case crossover design with a distributed lag nonlinear model (DLNM) was used by considering 27 lag days on CBVD admissions.

Results: Within five years (January 2014-December 2018), a total of 223,216 cases for cerebrovascular disease were collected after excluding nonlocal admissions, of which 9,541 (4.27\%), 149,757 (67.09\%), 7,444 (3.33\%), 31,356 (14.05\%), and 23,912 (10.71\%) were hospitalized for hemorrhagic stroke, ischemic stroke, atherosclerosis, intracranial aneurysm, and apoplexy sequela, respectively. In short, male participants and elderly individuals ( $\geq 65$ years) accounted for $57.6 \%$ and $61.7 \%$, respectively. Generally, the cumulative exposure-response curve for temperature was almost inverted " $\mathrm{V}$ "-shaped, with a slight increase at concentrations of higher than $25^{\circ} \mathrm{C}$. The cumulative exposure response curve for relative humidity was almost linear. The cumulative exposureresponse curves for atmospheric pressure were V-shaped, which meant that both high and low concentrations of atmospheric pressure may increase the risk of cerebrovascular disease incidence among patients with T2D.
\end{abstract}

Conclusions: The results of this study indicate that both an increase and a decrease in temperature and atmospheric pressure had a significant association with the occurrence of cerebrovascular disease among co morbid patients with diabetes. This study provides evidence of the short term effects of metrological factors on hospital admissions of CBVD among T2D patients.

Keywords: Admission; Beijing; Cerebrovascular disease; Distributed lag nonlinear model; Metrological factors, Type 2 diabetes 


\section{INTRODUCTION}

Since 1990, cerebrovascular diseases (CBVD) remain a leading cause of death worldwide $[1,2]$. CBVD risk factors are usually related to lifestyle habits such as smoking, alcohol consumption, insufficient physical activity, high blood pressure and cholesterol, and obesity, but also environmental factors such as metrological factors and air pollution make a significant contribution to CBVD onsets [3]. In 2019, cerebrovascular diseases accounted for 8.7 million deaths worldwide which is equivalent to about $14.6 \%$ of all deaths [4]. China faces the greatest challenge from stroke in the world, accounting for 1.57 million deaths in 2018 [5,6], accounting for approximately one-quarter of the overall mortality [7].

The impacts of metrological factors have been documented in worldwide studies [8,9] and studies carried on in North America (Schwartz, Samet et al. 2004, Anderson and Bell 2009, Goldberg, Gasparrini et al. 2011), Europe [10,11] and Australia [12], with a significant effect of heat waves on mortality and morbidity. Most previous studies have been studied about the effects of weather conditions on incidence of stroke which was not complicated with type 2 diabetes (T2D) and were conducted in developed countries [13,14]. However, the disease burden of co morbidities has become a serious issue for public health in developing countries like China. The short-term effects of metrological factors on cerebrovascular disease onset among diabetic participants in China remain largely unknown. Additionally, these associations at the city-specific level have not been thoroughly investigated, particularly in Beijing.

Therefore, in this study, we aimed to determine the acute effects of metrological factors on the hospitalization of causespecific CBVD among T2D patients, including hemorrhagic stroke, ischemic stroke, atherosclerosis, intracranial aneurysm and apoplexy sequela in Beijing, China.

\section{MATERIALS AND METHODS}

\section{Study area and data collection}

Beijing is located in the northern part of China at a latitude of $39 " 26^{\prime \prime}-41^{\prime \prime} 03^{\prime} \mathrm{N}$. The data recording system in the study area has been proven to be of high validity and good representativeness, covering more than $95 \%$ of medical services for permanent residents in Beijing [15]. The geographic locations of 258 hospitals included in the analysis have been described in our previous study [16].

Cerebrovascular admissions data were obtained from the Beijing Municipal Commission of Health and Family Planning Policy Research Center (http://xxzx.bjchfp.gov.cn/ hangyexinxi/) from January 1, 2014, to December 31, 2018.
The data were collected from 258 large general hospitals. Data included the patient's date of admission, principal diagnosis, age and sex. CBVD data were categorized according to the International $10^{\text {th }}$ Revision (ICD-10) codes to identify hemorrhagic stroke (ICD-10: I60-I62), ischemic stroke (ICD10: I63), atherosclerosis (ICD-10: I65), intracranial aneurysm (ICD-10: I67) and apoplexy sequela (ICD-10: I69) which were considered the primary outcomes. A history of T2D was coded as E12 and was defined as having fasting plasma glucose $\geq 7.1 \mathrm{mmol} / \mathrm{L}$ and/or current treatment of diabetes with ant diabetic medication before admission. According to the living address of cases, only cerebrovascular disease admissions among residents living in Beijing were included in this analysis.

Meteorological data was extracted from the China Meteorological Data Sharing Service System (https://data. cma.cn/en). Daily air pollution data was collected from 35 fixed-site air quality monitoring stations of the Beijing Municipal Environmental Protection Bureau (http://www. bjepb.gov.cn/) between January 1, 2014, and December 31, 2018.

\section{Ethical clearance}

In this study, informed consent was not specifically required because we only analyzed the aggregated data, which were typically used for administrative purposes we did not use personal data identifiers. However, the study was approved by the Institutional Review Board of Capital Medical University with the IRB00009511 identification number.

\section{Statistical analysis}

Statistical Analysis for continuous variables were described with mean \pm standard deviation (SD) or median and interquartile range (IQR). Categorical variables were presented as numbers and percentages. A time-stratified casecrossover design was adopted to remove control confounders and long-term trends [17]. Based on bidimensional functions called "cross-basis",

DLNM can reflect both lag structure of the association and the nonlinear exposure responses [18]. Therefore, the combination of the case-crossover design with DLNM was applied to simultaneously estimate the short-term, nonlinear, and lagged effects of metrological factors on cerebrovascular disease onset. Daily numbers of cerebrovascular diseases onset were modeled using generalized linear Poisson regression models to study the association between CBVD onset and meteorological variables including air temperature (mean, maximum, minimum, diurnal variation), atmospheric pressure (mean, maximum, minimum, diurnal variation), and 
relative humidity. Each meteorological variable was modeled separately after adjusting for day of week, public holiday (binary variable) and AQI, removing the confounding effect of those factors on CBVD onset. Modeling was also repeated in the subgroup analysis ( $<65$ years vs. $>65$ years, male vs. female). $\mathrm{Z}$ tests were used to compare differences between effect estimates in the strata [19]. The statistical tests were twosided, and $\mathrm{P}<0.05$ was considered statistically significant. All statistical analyses were conducted in R software (version 4.0.2) using the mgcv package

In our study, a DLNM model combined with a time-stratified case-crossover design was built as follows:

$Y t \sim \operatorname{Poisson}(\mu t)$

$(\mu t)=\alpha+($ Pollutanti, 3) + PCAi $+c b($ Tempt, 3$)+$ $c b($ RHt, 3$)+\mathrm{cb}($ atmt, 3$)+\gamma$ Holidayt $+\lambda$ Stratum

where $t$ is the specific day of observation; $Y t$ is the number of cerebrovascular cases on $t ; \mu t$ is the expectation of $Y t ; \alpha$ is the intercept; Pollutanti, $t$, Tempt, atmt and $R H t$ are, respectively, the $i t$ concentration of gaseous pollutant, daily temperature, daily atmospheric pressure and relative humidity on $t^{\prime} ; c b()$ is the cross-basis function for exposure-response natural spline for gaseous pollutant, daily temperature or relative humidity. Holiday is the indicator variable used to control the effect of public holidays, and Stratum represents the time stratum of the case-crossover design.

\section{RESULTS}

A total of 223,216 cases for cerebrovascular disease were collected after excluding nonlocal admissions, of which 9,541 (4.27\%), 149,757 (67.09\%), 7,444 (3.33\%),31,356 (14.05\%), and $23,912(10.71 \%)$ were hospitalized for hemorrhagic stroke, ischemic stroke, atherosclerosis, intracranial aneurysm, and apoplexy sequela, respectively. In short, male participants and elderly individuals ( $\geq 65$ years) accounted for $57.6 \%$ and $61.7 \%$, respectively. Table 1 summarizes the characteristics and distribution of daily cerebrovascular disease (overall and cause-specific), stratified by sex and age over the study period. During the study period, the median daily temperature, the median daily atmospheric pressure and the mean relative humidity were $14.4^{\circ} \mathrm{C}, 1012 \mathrm{mbar}$ and $54.3 \pm 19.2 \%$, respectively. Figure 1 presents a correlation analysis between atmospheric pollutants and meteorological factors using Pearson correlation test. The correlations between all pollutant concentrations and meteorological parameters (temperature and relative humidity) were calculated. Gaseous pollutants except O3 were strongly correlated with each other (coefficients ranged from 0.49 to 0.87 ) and moderately correlated with relative humidity and temperature (coefficients ranged from -0.41 to 0.33 ).

Figure 2 presents the relative risks of metrological factors (temperature, relative humidity and atmospheric pressure) on overall and cause-specific cerebrovascular diseases using different lag structures. The maximum relative risk (RR) was 1.006 (95\% CI: 1.004-1.007) for total CBVD, 1.005 (95\% CI: 1.003-1.006) for ischemic stroke, 1.007 (95\% CI: 1.0051.01) for atherosclerosis, 1.006 (95\% CI: 1.003-1.007) for intracranial aneurysm, and 1.008 (95\% CI: 1.006-1.011) for apoplexy sequela. Extremely high and low temperatures were associated with an increased risk of overall cerebrovascular cases (RR: 1.002; 95\% CI: 1.001-1.003) atlag1 day, intracranial aneurysm cases (RR: 1.003; 95\% CI: 1.002-1.004) at lag4 day, and apoplexy sequela cases (RR: 1.004; 95\% CI: 1.003-1.005) at lag0 day (Figure 2a). Positive associations were observed between relative humidity and risk for overall and cause specific cerebrovascular cases except ischemic stroke (Figure 2b). Both rapid increases and decreases in atmospheric pressure were associated with an increased risk of overall

Table 1: Characteristics and distribution of daily cerebrovascular cases in Beijing (2014-2018).

\begin{tabular}{|c|c|c|c|c|c|c|c|c|c|c|}
\hline & \multicolumn{2}{|c|}{ Total } & \multicolumn{2}{|c|}{ Male } & \multicolumn{2}{|c|}{ Female } & \multicolumn{2}{|c|}{$<65 \mathrm{y}$} & \multicolumn{2}{|c|}{$\geq 65 \mathrm{y}$} \\
\hline & $\mathbf{N}$ & P25-P75 & $\mathbf{N}(\%)$ & P25-P75 & N (\%) & P25-P75 & $\mathbf{N}(\%)$ & P25-P75 & $\mathbf{N}(\%)$ & P25-P75 \\
\hline Hemorrhagic stroke & 9541 & $3-7$ & $\begin{array}{c}5692 \\
(59.7)\end{array}$ & $2-4$ & $\begin{array}{c}3848 \\
(40.3)\end{array}$ & $1-3$ & $\begin{array}{c}4218 \\
(44.7)\end{array}$ & $1-3$ & $\begin{array}{c}5323 \\
(55.3)\end{array}$ & $2-4$ \\
\hline Ischemic stroke & 149757 & $52-104$ & $\begin{array}{l}87285 \\
(58.2) \\
\end{array}$ & $30-61$ & $\begin{array}{l}62469 \\
(41.8)\end{array}$ & $23-44$ & $\begin{array}{l}57454 \\
(38.2)\end{array}$ & $21-40$ & $\begin{array}{l}92303 \\
(61.8)\end{array}$ & $31-65$ \\
\hline Atherosclerosis & 7444 & $1-6$ & $\begin{array}{l}5460 \\
(73.2)\end{array}$ & $1-4$ & $\begin{array}{c}1984 \\
(26.8)\end{array}$ & $0-2$ & $\begin{array}{c}3412 \\
(45.3)\end{array}$ & $0-3$ & $\begin{array}{c}4032 \\
(54.7)\end{array}$ & $1-3$ \\
\hline Intracranial aneurysm & 31356 & $11-22$ & $\begin{array}{l}14876 \\
(47.4)\end{array}$ & $5-11$ & $\begin{array}{l}16480 \\
(52.6)\end{array}$ & $6-12$ & $\begin{array}{l}13068 \\
(41.6)\end{array}$ & $4-10$ & $\begin{array}{l}18288 \\
(58.4)\end{array}$ & $6-13$ \\
\hline Apoplexy sequela & 23912 & $3-21$ & $\begin{array}{l}14550 \\
(61.0)\end{array}$ & $2-13$ & $\begin{array}{c}9361 \\
(39.0)\end{array}$ & $1-8$ & $\begin{array}{c}7049 \\
(30.5)\end{array}$ & $1-6$ & $\begin{array}{l}16863 \\
(69.5)\end{array}$ & $2-15$ \\
\hline Overall & 223216 & $72-157$ & $\begin{array}{c}128508 \\
(57.6)\end{array}$ & $41-91$ & $\begin{array}{l}94703 \\
(42.4)\end{array}$ & $32-66$ & $\begin{array}{l}85726 \\
(38.3)\end{array}$ & $30-60$ & $\begin{array}{c}137490 \\
(61.7)\end{array}$ & $43-98$ \\
\hline
\end{tabular}




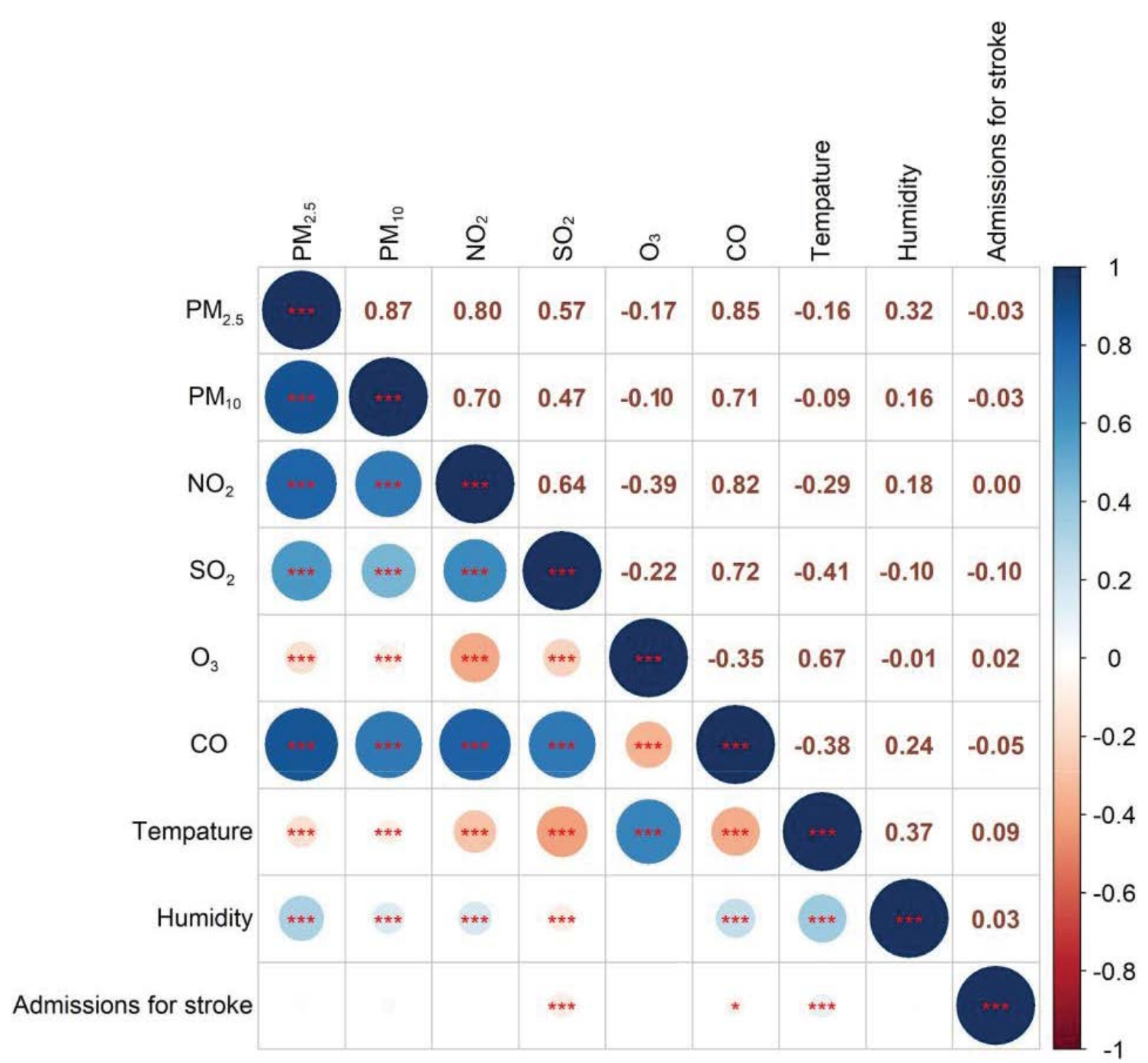

Figure 1: Pearson correlation coefficients between weather conditions and air pollution and daily admissions for cerebrovascular Disease in Beijing, 2014-2018 $(\mathrm{n}=1799)$.

cerebrovascular cases (RR: 1.0101; 95\% CI: 1.0099-1.0104) at lag0 day, hemorrhagic stroke cases (RR: 1.0386; 95\% CI: 1.0373-1.040) at lag0 day, ischemic stroke cases(RR: 1.003; 95\% CI: 1.0026-1.0033) at lag0 day, atherosclerosis cases (RR: 1.0017; 95\%CI: 1.0002-1.0032) at lag5 day, and intracranial aneurysm cases (RR: 1.0441; 95\% CI:1.0434-1.0449) at lag0 day (Figure 2c).

When temperature was $14.4^{\circ} \mathrm{C}$, a $20^{\circ} \mathrm{C}$ increase was associated with an increase of 1.0023 in the relative risk (RR) (95\% CI: 1.0011-1.0033) of hospital admissions for total CBVD, 1.0052 in the RR (95\% CI: 1.0023-1.0066) for Ischemic stroke, 1.0043 in the RR (95\% CI: 1.0033-1.0065) for Hemorrhagic stroke, and 1.0062 in the RR (95\%CI: 1.0044$1.0075)$ for Atherosclerosis. When relative humidity was 54.3 , a $20 \%$ increase was associated with a slight increase of 1.0132 in the RR (95\% CI: 1.0094-1.0145) for total CBVD and
1.0023 in the RR (95\% CI: 1.0013-1.0047) for Hemorrhagic stroke. However, no statistically significant association with relative humidity was observed for Ischemic stroke and Atherosclerosis admissions. When atmospheric pressure was 1012 mbar, a $20 \%$ increase was associated with an increase of 1.0011 in the RR (95\% CI: 1.0009-1.0014) for total CBVD, 1.0086 in the RR (95\% CI: 1.0073-1.0090) for Ischemic stroke, 1.0032 in the RR (95\% CI: 1.0026-1.0034) for Hemorrhagic stroke and 1.0017 in the RR 1.0017 (95\% CI: 1.0002-1.0032) for Atherosclerosis (Table 2).

As shown in Figure 3, the cumulative exposure-response curves of the overall association between weather conditions (temperature, relative humidity and atmospheric pressure) and cerebrovascular cases amongT2D patients were almost nonlinear. Generally, the cumulative exposure-response curve for temperature was almost inverted "V"-shaped, with 


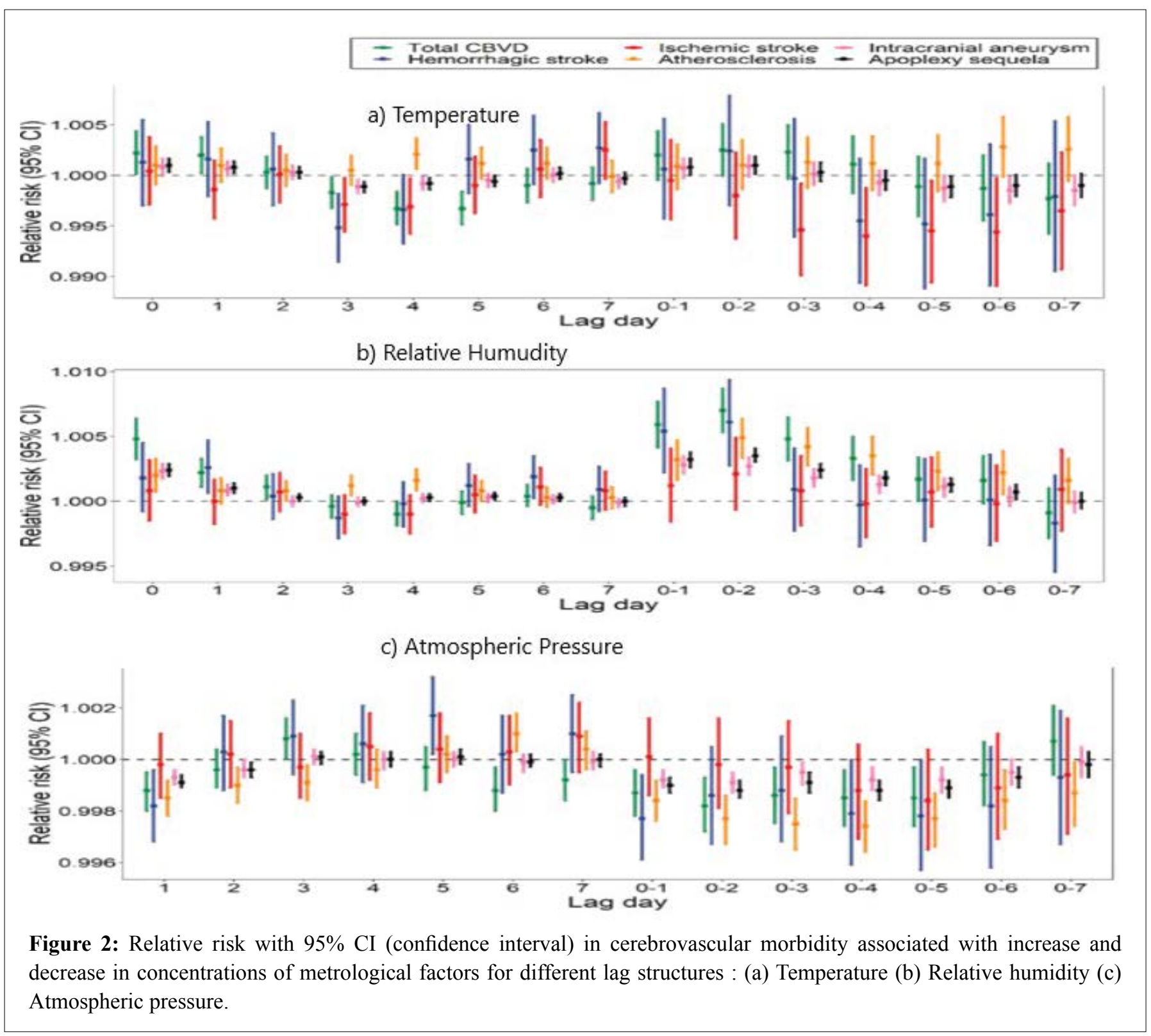

Table 2: RRs of event occurrence for temperature and relative humidity.

\begin{tabular}{|l|c|c|c|}
\hline \multirow{2}{*}{ Events } & \multicolumn{3}{|c|}{ RR 95\% CI } \\
\cline { 2 - 4 } & Temperature & Relative Humidity & Atmospheric pressure \\
\hline Total CBVD & $1.0023(1.0011-1.0033)$ & $1.0132(1.0094-1.0145)$ & $1.0011(1.0009-1.0014)$ \\
\hline Ischemic stroke & $1.0052(1.0023-1.0066)$ & $1.0023(1.0013-1.0047)$ & $1.0086(1.0073-1.0090)$ \\
\hline Hemorrhagic stroke & $1.0043(1.0033-1.0065)$ & $1.0000(1.0009-1.0019)$ & \\
\hline Atherosclerosis & $1.0062(1.0044-1.0075)$ & $1.0004(1.0002-1.0005)$ & \\
\hline
\end{tabular}

a slight increase at concentrations of higher than $25^{\circ} \mathrm{C}$. The cumulative exposure response curve for relative humidity was almost linear. The cumulative exposure-response curves for atmospheric pressure were $\mathrm{V}$-shaped, which meant that both high and low concentrations of atmospheric pressure may increase the risk of cerebrovascular disease onset among patients with T2D.

Table 3 Summary of the cumulative cold and hot effects associated with the 1st and 99.9th percentiles of temperature on the acute effects of CBVDs at lag 0, lag 5, lag 15 and lag 27. The highest hospitalization for all causes of specific CBVD occurred at lag 0 due to cold. 

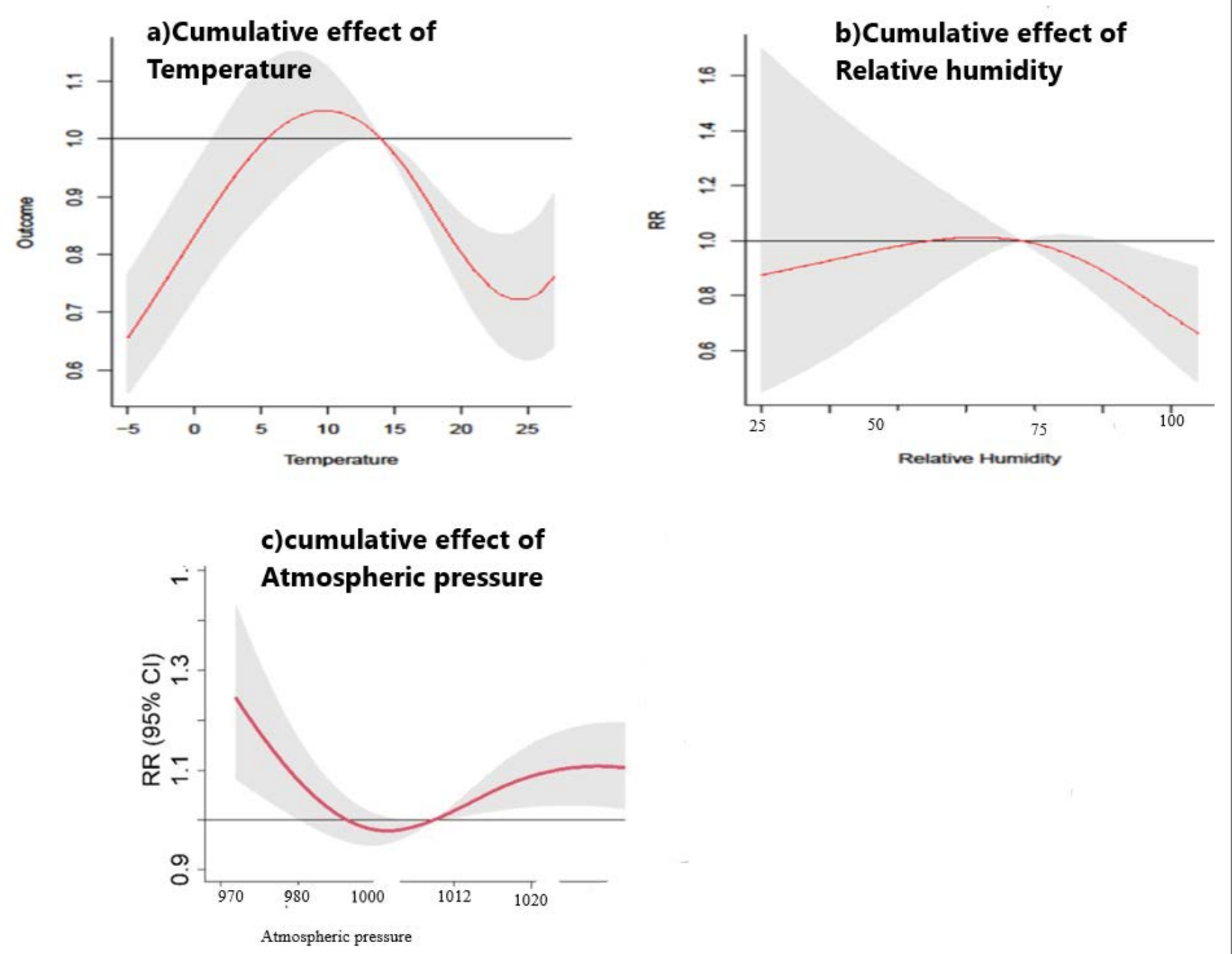

Figure 3: Cumulative exposure-response curves on overall cerebrovascular cases for metrological factors (temperature, relative humidity and atmospheric pressure):a) cumulative effect of temperature b) cumulative effect of relative humidity c) cumulative effect of atmospheric pressure

The distribution of meteorological factors and the counts of daily admissions for CBVD across four seasons from 2014 to 2018 are described in Figure S1.

\section{DISCUSSION}

This study was the first study to examine the acute effects of metrological factors on the hospitalization of people with CBVD among type 2 DM patients in Beijing, China. We found significant lagged and non linear effects of metrological factors (temperature, relative humidity and atmospheric pressure) on CBVD admission among T2D patients. In subgroup analysis, the associations were more significant in the elderly (age $>65$ years old) and male participants. In previous studies, metrological factors were associated with the incidence of cerebrovascular disease but haven't been studied among co morbid patients with diabetes. Our study added to the limited evidence for the risk of co morbidities of diabetes and cerebrovascular diseases due to the effect of metrological factors in developing countries like China.

The pathophysiologic effects of metrological factors on cerebrovascular disease among co morbid patients with T2D can be explained. Temperature changes induce blood vessels to dilate and constrict, which increase myocardial oxygen demand, myocardial ischemia, the cardiac work load and causes heart failure and may trigger cerebrovascular disease [20]. Atmospheric pressure may directly influence vessels walls by triggering endogenous inflammory and changing endothelial function $[21,22]$. 


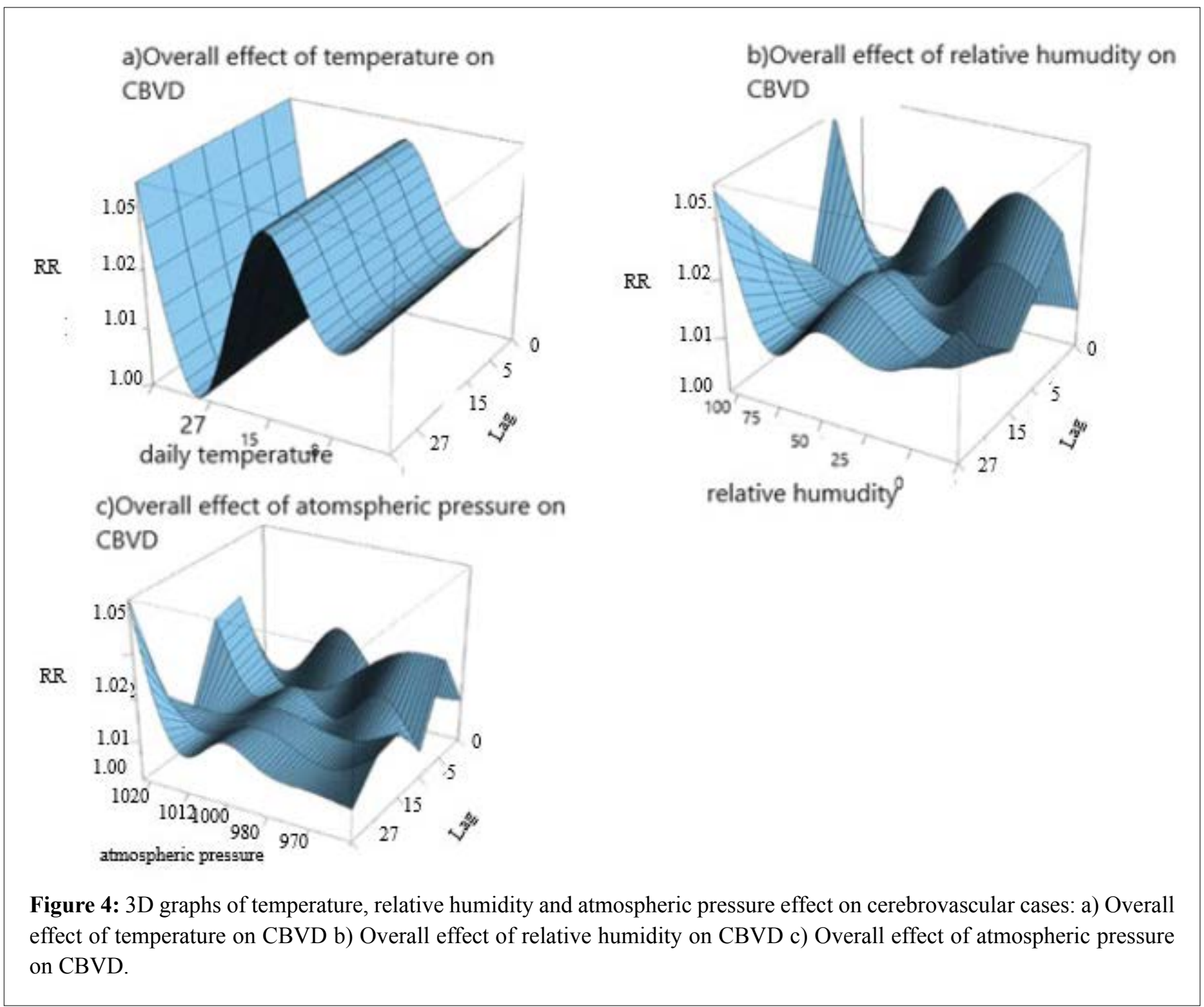

Table 3: The cumulative hot and cold effects (relative risks and 95\% CI) on cause-specific cerebrovascular diseases, including CBVD, Ischemic Stroke, Hemorrhagic Stroke and Atherosclerosis.

\begin{tabular}{|l|c|c|c|c|c|}
\hline Season effects & Lag & CBVD & Ischemic Stroke & Hemorrhagic Stroke & Atherosclerosis \\
\hline Hot effects & Lag 0 & $0.97(0.87,1.05)$ & $0.96(0.86,1.05)$ & $1.07(0.89,1.27)$ & $0.93(0.82,1.04)$ \\
\hline & Lag 5 & $1.056(1.01,1.07)$ & $1.05(0.89,1.27)$ & $1.08(1.01,1.12)$ & $1.038(1.00,1.07)$ \\
\hline & Lag 15 & $1.048(1.02,1.05)$ & $1.05(1.02,1.06)$ & $1.05(1.01,1.08)$ & $1.026(1.00,1.04)$ \\
\hline & Lag 27 & $1.06(1.03,1.08)$ & $1.06(1.03,1.09)$ & $1.12(1.07,1.18)$ & $1.03(0.98,1.05)$ \\
\hline Cold effects & Lag 0 & $1.17(1.04,1.27)$ & $1.04(0.52,1.29)$ & $1.10(0.89,1.33)$ & $1.04(0.91,1.16)$ \\
\hline & lag 5 & $0.95(0.89,0.95)$ & $0.92(0.88,0.94)$ & $0.95(0.88,1.00)$ & $0.99(0.94,1.02)$ \\
\hline & Lag 15 & $0.97(0.96,1.00)$ & $0.99(0.97,1.00)$ & $0.95(0.9,0.97)$ & $0.99(0.96,1.00)$ \\
\hline & Lag 27 & $0.95(0.90,0.97)$ & $0.96(0.90,0.97)$ & $1.00(0.92,1.06)$ & $0.96(0.91,1.00)$ \\
\hline
\end{tabular}

Most previous studies were designed to study the effects of metrological factors on the morbidity or mortality of stroke [23-25]. However, no existing study has examined the effects of metrological factors on cerebrovascular disease among patients with T2D. Many researchers have described
U-, V- or J-shaped associations between meteorological factors and cerebrovascular mortality [24,26]. Similarly, the present study found the cumulative exposure-response curve for temperature was almost inverted "V"-shaped, with a slight increase at concentrations of higher than $25^{\circ} \mathrm{C}$. The 
cumulative exposure response curve for relative humidity was almost linear. The cumulative exposure-response curves for atmospheric pressure were V-shaped, which meant that both high and low concentrations of atmospheric pressure may increase the risk of cerebrovascular disease onset among patients with T2D.

There are some strengths of our study. First, the data on cerebrovascular cases has been proven to be of high validity and good representativeness, covering more than 95\% of permanent residents in Beijing, and the sample size was relatively large. Therefore, the present results may be representative of the authentic associations between weather conditions and cerebrovascular diseases in the study area. Second, this study was the first time that a nonlinear and lagged association was observed between metrological factors and morbidity of cerebrovascular diseases in a population with T2D, which may have important implications for the prevention and treatment of CBVD.

The study had also some limitations. First, we could not collect data on the relationships between patients. However, there might be a few family aggregation cases, which may affect the population sensitivity [27]. Second, we used fixedsite monitor measurements as a proxy for personal exposure, which may result in exposure errors and underestimation of the associations between weather conditions and diseases. Third, since the date of diagnostic is not available, it cannot be differentiated between the accurate occurrence of stroke and T2D. Fourth, the generalizability of our results might be limited as the study collected data from one highly polluted city and only included T2D [28-36].

\section{CONCLUSIONS}

Our study suggested that both an increase and a decrease in temperature and atmospheric pressure had a significant association with the occurrence of cerebrovascular disease among co morbid patients with diabetes. This study provides evidence of the short term effects of metrological factors on hospital admissions of CBVD among T2D patients

\section{ABBREVIATIONS}

CBVD: Cerebrovascular Disease; ICD: International Classification of Disease; T2D: Type 2 Diabetes; PM2.5: Fine Particulate Matter with an Aerodynamic Diameter; PM10: Inhalable Particulate Matter with an Aerodynamic Diameter; NO2: Nitrogen Dioxide; SO2: Sulfur Dioxide; O3: Ozone; 95\% CI: 95\% Confidence Interval; CO: Carbon Monoxide; GAM: Generalized Additive Model; df: Degrees of Freedom; SD: Standard Deviation; IQR: Interquartile Range

\section{ACKNOWLEDGEMENT}

We acknowledge the Beijing Municipal Health Commission Information Center andthe Beijing Air Pollution and Metrological Data Center.

\section{FUNDING}

This study was supported by National Natural Science Foundation of China (No. 82003559), Nature Science Foundation of Capital Medical University (No. PYZ2018046), Beijing Municipal Training Project of Excellent Talents. The funding was neither used for the study design nor data collection but to cover for the publication fees.

\section{AVAILABILITY OF DATA AND MATERIALS}

The data can be accessed from the Beijing Municipal Health Commission Information Center with permission via direct request.

\section{AUTHORS' CONTRIBUTIONS}

Deginet Aklilu: Conceptualization, methodology, formal analysis. investigation, data curation, writing original draft, writing review and editing. Tianqi Wang: Conceptualization, data curation. AntenehTamirat: methodology, formal analysis. FengiWei: Conceptualization, methodology Zhiwei Li: methodology, formal analysis. Xia Li: Conceptualization, methodology. Lixin Tao: data curation. Xiangtong Liu: methodology, formal analysis. MoningGuo: methodology, formal analysis. WeiWang: Conceptualization, methodology. XiuhuaGuo: Conceptualization, methodology, data curation, writing review and editing.

\section{ETHICS APPROVAL}

In this study, informed consent was not specifically required because we did not use personal data identifiers. However, the study was approved by the Institutional Review Board of Capital Medical University with the IRB00009511 identification number.

\section{CONSENT FOR PUBLICATION}

Not applicable.

\section{COMPETING INTERESTS}

The authors declare that they have no competing interests.

\section{REFERENCES}

1. Murray, C. J. L., A. D. Lopez (1996) The global burden of disease: a comprehensive assessment of mortality and disability from diseases, injuries, and risk factors in 1990 and projected to 2020. Cambridge, Mass. Published by the Harvard School of Public Health on behalf of the World Health Organization and the World Bank. 
2. Feigin, V. L., B. Norrving, G. A. Mensah (2017) Global Burden of Stroke. Circ Res 120(3): 439-448.

3. Chen, R., C. Wang, X. Meng, H. Chen, T. Q. Thach, C. M. Wong, H. Kan (2013) Both low and high temperature may increase the risk of stroke mortality. Neurology 81(12): 1064-1070.

4. Virani, S. S., A. Alonso, H. J. Aparicio, E. J. Benjamin, M. S. Bittencourt, C. W. Callaway, A. P. Carson, A. M. Chamberlain, S. Cheng, F. N. Delling, M. S. V. Elkind, K. R. Evenson, J. F. Ferguson, D. K. Gupta, S. S. Khan, B. M. Kissela, K. L. Knutson, C. D. Lee, T. T. Lewis, J. Liu, M. S. Loop, P. L. Lutsey, J. Ma, J. Mackey, S. S. Martin, D. B. Matchar, M. E. Mussolino, S. D. Navaneethan, A. M. Perak, G. A. Roth, Z. Samad, G. M. Satou, E. B. Schroeder, S. H. Shah, C. M. Shay, A. Stokes, L. B. VanWagner, N.-Y. Wang, C. W. Tsao (2021) Heart Disease and Stroke Statistics\&\#x2014;2021 Update. Circulation 143(8): e254-e743.

5. Wang, W., B. Jiang, H. Sun, X. Ru, D. Sun, L. Wang, L. Wang, Y. Jiang, Y. Li, Y. Wang, Z. Chen, S. Wu, Y. Zhang, D. Wang, Y. Wang, V. L. Feigin (2017) Prevalence, Incidence, and Mortality of Stroke in China: Results from a Nationwide Population-Based Survey of 480687 Adults. Circulation 135(8): 759-771.

6. Wang, Y. J., Z. X. Li, H. Q. Gu, Y. Zhai, Y. Jiang, X. Q. Zhao, Y. L. Wang, X. Yang, C. J. Wang, X. Meng, H. Li, L. P. Liu, J. Jing, J. Wu, A. D. Xu, Q. Dong, D. Wang, J. Z. Zhao (2020) China Stroke Statistics 2019: A Report From the National Center for Healthcare Quality Management in Neurological Diseases, China National Clinical Research Center for Neurological Diseases, the Chinese Stroke Association, National Center for Chronic and Noncommunicable Disease Control and Prevention, Chinese Center for Disease Control and Prevention and Institute for Global Neuroscience and Stroke Collaborations. Stroke Vasc Neurol 5(3): 211-239.

7. Zhou, M., H. Wang, J. Zhu, W. Chen, L. Wang, S. Liu, Y. Li, L. Wang, Y. Liu, P. Yin, J. Liu, S. Yu, F. Tan, R. M. Barber, M. M. Coates, D. Dicker, M. Fraser, D. González-Medina, H. Hamavid, Y. Hao, G. Hu, G. Jiang, H. Kan, A. D. Lopez, M. R. Phillips, J. She, T. Vos, X. Wan, G. Xu, L. L. Yan, C. Yu, Y. Zhao, Y. Zheng, X. Zou, M. Naghavi, Y. Wang, C. J. Murray, G. Yang, X. Liang (2016) Cause-specific mortality for 240 causes in China during 1990-2013: a systematic subnational analysis for the Global Burden of Disease Study 2013. Lancet 387(10015): 251-272.

8. Barnett, A. G., A. J. Dobson, P. McElduff, V. Salomaa, K. Kuulasmaa, S. Sans (2005) Cold periods and coronary events: an analysis of populations worldwide. Journal of Epidemiology and Community Health 59(7): 551-557.

9. McMichael, A. J., P. Wilkinson, R. S. Kovats, S. Pattenden, S. Hajat, B. Armstrong, N. Vajanapoom, E. M. Niciu, H. Mahomed, C. Kingkeow, M. Kosnik, M. S. O’Neill, I. Romieu, M. Ramirez-Aguilar, M. L. Barreto, N. Gouveia, B. Nikiforov (2008) International study of temperature, heat and urban mortality: the 'ISOTHURM' project. International Journal of Epidemiology 37(5): 1121-1131.

10. Belmin, J., J.-C. Auffray, C. Berbezier, P. Boirin, S. Mercier, B. de Reviers, J.-L. Golmard (2007) Level of dependency: a simple marker associated with mortality during the 2003 heatwave among French dependent elderly people living in the community or in institutions. Age and Ageing 36(3): 298-303.
11. Mastrangelo, G., U. Fedeli, C. Visentin, G. Milan, E. Fadda, P. Spolaore (2007) Pattern and determinants of hospitalization during heat waves: an ecologic study. BMC Public Health 7: 200.

12. Wang, X. Y., A. G. Barnett, W. Yu, G. FitzGerald, V. Tippett, P. Aitken, G. Neville, D. McRae, K. Verrall, S. Tong (2012) The impact of heatwaves on mortality and emergency hospital admissions from non-external causes in Brisbane, Australia. Occupational and Environmental Medicine 69(3): 163-169.

13. Gunes, H., H. Kandis, A. Saritas, S. Dikici, R. Buyukkaya (2015) The relationship between ischemic stroke and weather conditions in Duzce, Turkey. World journal of emergency medicine 6(3): 207-211.

14. Vencloviene, J., R. Radisauskas, D. Kranciukaite-Butylkiniene, A. Tamosiunas, V. Vaiciulis, D. Rastenyte (2021) Association between stroke occurrence and changes in atmospheric circulation. BMC Public Health 21(1): 42.

15. Aklilu, D., T. Wang, E. Amsalu, W. Feng, Z. Li, X. Li, L. Tao, Y. Luo, M. Guo, X. Liu, X. Guo (2020) Short-term effects of extreme temperatures on cause specific cardiovascular admissions in Beijing, China. Environmental Research 186: 109455.

16. Li, H., J. Wu, A. Wang, X. Li, S. Chen, T. Wang, E. Amsalu, Q. Gao, Y. Luo, X. Yang, W. Wang, J. Guo, Y. Guo, X. Guo (2018) Effects of ambient carbon monoxide on daily hospitalizations for cardiovascular disease: a time-stratified case-crossover study of 460,938 cases in Beijing, China from 2013 to 2017. Environmental Health 17(1): 82.

17. Liu, L., F. Song, J. Fang, J. Wei, H. C. Ho, Y. Song, Y. Zhang, L. Wang, Z. Yang, C. Hu, Y. Zhang (2021) Intraday effects of ambient $\mathrm{PM}(1)$ on emergency department visits in Guangzhou, China: A case-crossover study. Sci Total Environ 750: 142347.

18. Gasparrini A (2014) Modeling exposure-lag-response associations with distributed lag non-linear models. Stat Med 33(5): 881-899.

19. Lin, H., T. Liu, J. Xiao, W. Zeng, X. Li, L. Guo, Y. Zhang, Y. Xu, J. Tao, H. Xian, K. M. Syberg, Z. Qian, W. Ma (2016) Mortality burden of ambient fine particulate air pollution in six Chinese cities: Results from the Pearl River Delta study. Environment International 96: 91-97.

20. Tian, Y., H. Liu, Y. Si, Y. Cao, J. Song, M. Li, Y. Wu, X. Wang, X. Xiang, J. Juan, L. Chen, C. Wei, P. Gao, Y. Hu (2019) Association between temperature variability and daily hospital admissions for cause-specific cardiovascular disease in urban China: A national time-series study. PLOS Medicine 16(1): e1002738.

21. Cao, Y., X. Wang, D. Zheng, T. Robinson, D. Hong, S. Richtering, T. H. Leong, A. Salam, C. Anderson, M. L. Hackett (2016) Air Pressure, Humidity and Stroke Occurrence: A Systematic Review and Meta-Analysis. Int J Environ Res Public Health 13(7).

22. Guan, W., S. J. Clay, G. J. Sloan, L. G. Pretlow (2018) Effects of Barometric Pressure and Temperature on Acute Ischemic Stroke Hospitalization in Augusta, GA. Transl Stroke Res.

23. Dawson, J., C. Weir, F. Wright, C. Bryden, S. Aslanyan, K. Lees, W. Bird, M. Walters (2008) between meteorological variables and acute stroke hospital admissions in the west of Scotland. Acta Neurol Scand 117(2): 85-89. 
24. Jimenez-Conde, J., A. Ois, M. Gomis, A. Rodriguez-Campello, E. Cuadrado-Godia, I. Subirana, J. Roquer (2008) Weather as a trigger of stroke. Daily meteorological factors and incidence of stroke subtypes. Cerebrovasc Dis 26(4): 348-354.

25. 25 Nocera, R., P. Petrucelli, J. Park, E. Stander (2014) Meteorological Variables Associated with Stroke. International Scholarly Research Notices 2014: 597106.

26. Turner, L. R., D. Connell, S. Tong (2013) The Effect of Heat Waves on Ambulance Attendances in Brisbane, Australia. Prehospital and Disaster Medicine 28(5): 482-487.

27. Yang, Y., Y. Guo, Z. Qian, Z. Ruan, Y. Zheng, A. Woodward, S. Ai, S. W. Howard, M. G. Vaughn, W. Ma, F. Wu, H. Lin (2018) Ambient fine particulate pollution associated with diabetes mellitus among the elderly aged 50 years and older in China. Environmental Pollution 243: 815-823.

28. Anderson, B. G., M. L. Bell (2009) Weather-related mortality: how heat, cold, and heat waves affect mortality in the United States. Epidemiology 20(2): 205-213.

29. Goldberg, M. S., A. Gasparrini, B. Armstrong, M.-F. Valois (2011) The short-term influence of temperature on daily mortality in the temperate climate of Montreal, Canada. Environmental Research 111(6): 853-860.

30. Lian, H., Y. Ruan, R. Liang, X. Liu, Z. Fan (2015) Short-Term Effect of Ambient Temperature and the Risk of Stroke: A
Systematic Review and Meta-Analysis. International Journal of Environmental Research and Public Health 12(8): 9068-9088.

31. Liu, X., Z. Li, M. Guo, J. Zhang, L. Tao, X. Xu, A. Deginet, F. Lu, Y. Luo, M. Liu (2021) Acute effect of particulate matter pollution on hospital admissions for stroke among patients with type 2 diabetes in Beijing, China, from 2014 to 2018. Ecotoxicology and Environmental Safety 217: 112201.

32. Mostofsky, E., E. H. Wilker, J. Schwartz, A. Zanobetti, D. R. Gold, G. A. Wellenius, M. A. Mittleman (2014) Short-term changes in ambient temperature and risk of ischemic stroke. Cerebrovascular diseases extra 4, 9-18 DOI: 10.1159/000357352.

33. Rothwell, P., S. Wroe, J. Slattery, C. Warlow (1996) Is stroke incidence related to season or temperature? The Oxfordshire Community Stroke Project. Lancet 347(9006): 934-936.

34. Schwartz, J., J. M. Samet, J. A. Patz (2004) Hospital admissions for heart disease: the effects of temperature and humidity. Epidemiology 15(6): 755-761.

35. Stang A (2004) Ischemic Stroke and Temperature Decrease. Epidemiology 15(1).

36. Wang, X., Y. Cao, D. Hong, D. Zheng, S. Richtering, E. C. Sandset, T. H. Leong, H. Arima, S. Islam, A. Salam, C. Anderson, T. Robinson and M. L. Hackett (2016) Ambient Temperature and Stroke Occurrence: A Systematic Review and Meta-Analysis. International journal of environmental research and public health 13(7): 698 . 